\title{
Effect of the Dome on Divertor Performance in ITER
}

\author{
A. S. Kukushkin ${ }^{\mathrm{a},{ }^{*}}$, H. D. Pacher ${ }^{\mathrm{b}}$, V. Kotov $^{\mathrm{c}}$, D. Reiter ${ }^{\mathrm{c}}$, D. P. Coster $^{\mathrm{d}}$, G. W. Pacher ${ }^{\mathrm{e}}$ \\ a ITER International Team, Garching Joint Work Site, Garching, Germany, \\ ${ }^{b}$ INRS-EMT, Varennes, Québec, Canada, \\ ${ }^{c}$ FZ Jülich, Jülich, Germany, ${ }^{d}$ Max-Planck Institut für Plasmaphysik, Garching, Germany, \\ ${ }^{e}$ Hydro_Québec, Varennes, Québec, Canada
}

\begin{abstract}
The effect of variation of the divertor geometry in ITER is re-evaluated with the recently developed non-linear model of the transport of the neutral particles, taking into account neutral-neutral and molecule-ion collisions. The importance of the "dome" for the neutral gas compression in the divertor is confirmed. Unlike earlier studies with a linear model of neutral transport, the present results show only a weak sensitivity of the divertor performance to the details of the divertor shape.
\end{abstract}

PSI17 keywords: B2/Eirene, Edge modelling, ITER, Divertor Geometry, Divertor Neutrals JNM keywords: T0100, P0600

PACS: 52.25.Ya, 52.65.Kj, 52.65.Pp

* Andrei S. Kukushkin, ITER IT, c/o Max-Planck Institut für Plasmaphysik, Boltzmannstr. 2, D-85748, Garching, Germany. E-mail: Andrei.Kukushkin@iter.org, tel. +49-89-3299-4122, fax +49-89-3299-4165 


\section{Introduction}

Continuing optimization of the design of the divertor cassettes for ITER involves reassessment of the role of the divertor "dome" and the effect of variations of its shape on divertor performance. Use of a recently improved neutral transport model which takes into account neutral-neutral and molecule-ion collisions [1] allows a comparative modelling analysis of the effect of dome variation, including optionally complete removal of the dome. First results of comparison between divertor operation with and without the dome were reported in [2]. Here, we refine the computational grid to avoid possible artifacts and thereby confirm the results of [2]. Furthermore, we explore possible design optimization by investigating the consequences of making the dome longer.

Since pumping is critical for helium removal but the pumping capability of ITER is limited, it is important to refine the definition of the pumping-related parameters in the framework of the improved model to determine a more precise relation between the plasma and the engineering parameters, as shown in section 2. Section 3 presents the results on the variation of dome length, and section 4 contains the conclusions.

We use the B2-Eirene code package, version solps4.2 [1], combining a full multi-fluid description of the electrons and $\mathrm{D}, \mathrm{He}$, and $\mathrm{C}$ ions, with a non-linear Monte-Carlo neutral model in toroidal geometry.

\section{Definition of Neutral Gas Parameters}

In previous studies, we determined that the most appropriate parameter to characterize the operating point in edge parameter space is the appropriately normalized average neutral pressure at the entrance to the private flux region, PFR (averaged over the outermost grid cells facing the PFR below the dome, Fig. 1a) [3]. Use of this parameter, $p_{D T}$, ensures a good parameterization of the modelling results $[4,5]$. The effective pumping speed at the grid edge 
was defined as $S_{D T}$, the ratio of pumping throughput $\Gamma_{\mathrm{DT}}$ in Pa $\cdot \mathrm{m}^{3} / \mathrm{s}$ to $p_{D T}$. In the past, this quantity was the only one that could be related to the actual pumping speed at the pump duct, since with the previous linear model the neutral pressure could be calculated only inside the computational grid used for the plasma transport. However, the neutral fluxes and energies on the surfaces in the PFR were unrealistic because of lack of collisions in the PFR and nonthermalised molecules, and moreover tended to exaggerate geometric effects, so that they could not be used for determining the neutral gas parameters in the PFR. The introduction of the new, non-linear neutral transport model allows now a more accurate estimation of the neutral gas parameters in the PFR from the neutral fluxes onto the surfaces there. Indeed, suppose that one-sided particle $\Gamma$ and energy $Q$ fluxes are calculated for a surface of the area S. Then, assuming Maxwellian distribution functions, one obtains for the neutral gas parameters in front of this surface

$$
T_{e V}=3.1 \cdot 10^{18} Q / \Gamma, n=2.6 \cdot 10^{-4} \sqrt{\frac{A}{T_{e V}}}\left(\frac{\Gamma}{S}\right), \quad p=4.1 \cdot 10^{-23} \sqrt{A T_{e V}}\left(\frac{\Gamma}{S}\right)
$$

Here $A$ is the mass number of the neutral particles, $n$ and $p$ the neutral density and pressure. $T_{e V}$ is the neutral temperature in $\mathrm{eV}$, and the other parameters are in MKS units.

The effective pumping speed $S_{D T}$ used in [1-6] is related, but not proportional, to the pumping speed at the pump duct. Indeed, the neutrals at the PFR interface have a high (several eV) energy whereas the engineering pumping speed is evaluated at room temperature. The gas conductance of the channel under the dome, which connects the PFR interface to the pump duct, is finite and this impedes the neutral flux to the pump. In our calculations, we model pumping by specifying a certain absorption coefficient $\xi$ for all neutral particles at the surface representing the duct entrance, Fig. 1a, and adjusting the $\xi$ value to obtain the desired $S_{D T}$. In reality, the ducts are toroidally discontinuous, they are part of the toroidal ring for which $\xi$ is specified in our 2D calculations, and our $\xi$ includes also the ratio $\gamma$ of the duct opening area to 
the total area of the ring. It was shown in [7] that the total pumping speed at the duct entrance does not depend on $\gamma$, once the absorption probability is specified in 2D geometry and $\xi<<\gamma$. Following the approach of [7], we find the expression for the real pumping speed at the duct entrance (in $\mathrm{m}^{3} / \mathrm{s}$ )

$$
c_{p}=3.9 \cdot 10^{3} \xi S \sqrt{\frac{T_{e V}}{A}} .
$$

Evaluating at room temperature, $T_{e V}=0.025 \mathrm{eV}$, one obtains for the "engineering” pumping speed $c_{p}^{\text {eng }}=6.2 \cdot 10^{2} A^{-\frac{1}{2}} S \xi$. The major result of this exercise is that, wherever we quoted an "effective" pumping speed for ITER of $S_{D T}=20 \mathrm{~m}^{3} / \mathrm{s}$ for the previous linear neutral model, the absorption and area correspond to an "engineering” pumping speed of $\sim 115 \mathrm{~m}^{3} / \mathrm{s}$ whereas the same effective pumping speed within the non-linear neutral model corresponds to an "engineering” pumping speed of $\sim 60 \mathrm{~m}^{3} / \mathrm{s}$. In becoming more collisional, the neutral gas becomes both more isotropic and cooler. This difference between the models is even larger for helium as shown in [8] - heating the molecules in collisions with plasma ions in the new model apparently provides a partial compensation of the effect on atoms.

\section{Variation of the Dome}

The primary function of the dome is to increase the neutral pressure in the PFR to make helium pumping more efficient. Besides this, it could be helpful in reducing the neutral reflux to the core through the x-point, in protecting diagnostic equipment in the PFR from plasma, and in neutron shielding of the pump duct. However, it adds to the complexity of the divertor cassette design and to the machine cost. Since most of the dome's functions are related to interaction with neutrals, whose modelling is improved with the nonlinear neutral model, we are led to re-assess the effect of the dome on the divertor performance with this model. The consequences of dome removal on the divertor performance of ITER were reported in [2]. The main effect was found to be a factor 3 increase of the pumping speed required to obtain 
the same conditions for helium removal. Indeed, without the dome, the plasma in the PFR absorbs neutrals more efficiently, and the helium atoms entering the PFR through the PFR interface region, Fig. 1a, have a lower probability of reaching the pump duct since many of them return to the plasma in the area previously shielded by the dome. In other words, the ratio of $c_{p}^{e n g} / S_{D T}$ would increase drastically if the dome were removed.

The calculations with and without dome in [2] were done on the same plasma grid, Fig. 1a. When the dome was removed, artificial plasma recycling occurred on the grid edges facing the PFR in the regions previously shielded by the dome. In order to estimate the effect of this recycling, a series of runs on a wider grid, limited in the PFR by the pump duct, Fig. 1b, was done for the case without dome. With this grid, the whole PFR area where the plasma flux is significant is modelled, the plasma fluxes on the grid edge in PFR are reduced by 3 to 4 orders of magnitude, and the artificial recycling and related release of carbon become negligible. The result is illustrated in Fig. 2 where the dependence of the peak power loading on the target $q_{p k}$ and separatrix density of helium ions $n_{\text {He_sep }}$ times normalized pumping speed $S_{\#}$ on the normalized neutral pressure in the PFR $\mu$ is compared between these two grid options and with the standard ITER geometry. Here the pressure is normalised to its value at the incipient detachment for each geometry, and $S_{\#}$ is $c_{p}^{e n g} / S_{0}$ where $S_{0}=115 \mathrm{~m}^{3} / \mathrm{s}$. This selection of parameters allows meaningful comparison in terms of the operational window of the divertor, and the helium related quantities, such as $n_{H e \_s e p}$ and the helium atom influx into the core $\Gamma_{\text {He_n_sep, }}$, are almost inversely proportional to the pumping speed [1, 3, 4]. After this normalization, divertor and core interface parameters are practically independent of the grid, and one can see that removal of the dome would result in expanding the operating window to $q_{p k}$ about a factor of 2 lower - the presence of the dome enhances the in-out asymmetry of the target loading and the window is limited by the detachment of the less loaded inner divertor, whereas the peak power load occurs at the outer divertor. However, the pumping speed 
needed to maintain the same helium density at the separatrix would have to be increased by a factor of 2 to 8 . The average energies of neutrals arriving at the duct entrance are significantly higher in the domeless configuration. This higher neutral temperature leads to an increase of the pumping speed as $T^{1 / 2}$, Eq. (2), but the density decreases for the same neutral pressure as $T^{-1}$ and therefore the engineering pumping speed has to be increased to ensure the same particle exhaust at the same pressure when the temperature increases. In other words, the dome compresses the neutrals not only by impeding their return to the plasma, but also by cooling them down.

Another variation on the dome theme is an increase of its size. Fig. 3a shows a typical profile of helium flux across the inner and outer outlets of the under-dome channel, from the divertor floor to the dome, positive towards the pump. Helium flows away from the pump in the upper part of this channel because its pressure across the channel from floor to dome is nearly uniform whereas the pressure inside the plasma in the PFR decreases rapidly with the distance from the targets, so that the pressure gradient reverses. One could thus expect the reduced height channel associated with a longer dome to result in more efficient helium removal. This can only be examined with the full nonlinear neutral model.

For this study, we selected a geometry with channel height a factor 2 smaller, Fig. 3b. Such a modification could also be advantageous from the technical point of view since it renders the dome and supporting structures more robust. The non-linear neutral transport model predicts [2] that the liner transparency $\zeta$ (roughly the toroidal fraction of the under-dome channel opening in the circumference) can be reduced to 0.1 (cf. 0.5 for the linear model [6]) before the divertor asymmetry is affected negatively, and that therefore a more robust divertor dome design is possible. Therefore we will first compare the standard ITER geometry, at $\zeta=0.1$, with the long dome, at $\zeta=0.2$, so as to modify the transparency but keep the same aperture 
of the channel, and then reduce $\zeta$ back to 0.1 to separate the effect of the dome length from that of the reduction of the channel conductance.

After the normalization described above, most of the plasma parameters at both the target and the separatrix follow the same dependence on $\mu$ (e.g., Fig. 4a), except $q_{p k}$, Fig. 4b, and helium-related quantities. As expected, the neutral pressure in the under-dome channel increased by about $40 \%$ for the same $\mu$, i.e. the same operating point relative to detachment, explaining the improved $n_{\text {He_sep }} \cdot S_{\#}$ values. Both the helium ion density at the separatrix and the helium atom influx decrease by about a factor 2 since the helium atoms released from PFR enter the plasma further from the x-point. No significant difference is seen between the two long dome options, suggesting that the threshold value of $\xi$ at which the liner transparency starts to play a role shifts down - apparently, the gas conductance in the channel due to the pressure increase [9] outweighs the reduction of the cross-section.

\section{Conclusions and Outlook}

The improved, non-linear model of the neutral particle transport now used in the B2-Eirene code package allows a more accurate evaluation of the neutral pressure at the pump duct. With this model, it is possible for the first time to relate the conditions at the edge of the plasma facing the PFR, which determine the operating point, to the engineering pumping speed. The results show that the conditions modelled correspond to a design value of pumping speed of $60 \mathrm{~m}^{3} / \mathrm{s}$, which is near the technical limit imposed by the pumping system design for ITER $\left(75 \mathrm{~m}^{3} / \mathrm{s}\right)$.

Analysis done with the refined computational grid confirms the importance of the divertor dome in ensuring the neutral gas compression which is beneficial for helium exhaust. Although removal of the dome would allow an expansion of the operational window down to about a factor 2 lower peak power loading of the target, this would require a pumping speed 
higher by an order of magnitude to maintain the same efficiency of helium removal throughout this window. Since the technical limits of ITER do not allow such an increase in pumping speed, the effect of higher helium concentration in the core on the reactor flexibility must be assessed, together with other concerns like divertor diagnostic access, neutron shielding, and so on, before a decision on the dome removal could be made. Since the ITER reference design is already expected to ensure a reasonably wide operational window in peak power loading, and the time scale for the divertor design decision is short, we do not recommend considering the dome removal as a viable design option.

Our present results obtained with the non-linear neutral model which predicts higher gas conductance between the inner and outer divertors, indicate that the requirement on the transparency of the dome-supporting structures can be relaxed, and that therefore the dome design can be made more reliable, with stronger supporting structures. An increase of the dome length would clearly improve the helium removal efficiency but this can be outweighed by the accompanying reduction of the operational window in power loading.

The results obtained with the non-linear model of neutral particle transport in the ITER divertor are much less sensitive to details of the geometry than those obtained from the linear model, which is still often used because of computational speed considerations. We are continuing re-assessment of our previous recommendations on the divertor design for ITER, which were based on the linear model, and first results indeed indicate that some geometrical restrictions can be relaxed (to be reported elsewhere).

\section{Acknowledgement}

This report was prepared as an account of work undertaken within the framework of ITER Transitional Arrangements (ITA). These are conducted by the Participants: China, the European Atomic Energy Community, India, Japan, Korea, the Russian Federation, and the 
United States of America, under the auspices of the International Atomic Energy Agency. The views and opinions expressed herein do not necessarily reflect those of the Participants to the ITA, the IAEA or any agency thereof. Dissemination of the information in this paper is governed by the applicable terms of the former ITER EDA Agreement. 


\section{References}

[1] A.S. Kukushkin, H.D. Pacher, V. Kotov, et al., Nucl. Fusion 45 (2005) 608.

[2] A.S. Kukushkin, H.D. Pacher, V. Kotov, et al., Proc. $32^{\text {nd }}$ EPS Conf. on Contr. Fusion and Plasma Phys., Tarragona, 2005.

[3] H.D. Pacher, A.S. Kukushkin, G.W. Pacher, et al., J. Nucl. Mater. 313-316 (2003) 657.

[4] A.S. Kukushkin, H.D. Pacher, G.W. Pacher et al., Nucl. Fusion 43 (2003) 716.

[5] A.S. Kukushkin, H.D. Pacher, D. Coster, et al., J. Nucl. Mater. 337-339 (2005) 50.

[6] A.S. Kukushkin, H.D. Pacher, Plasma Phys. Contr. Fusion 44 (2002) 931.

[7] S.I. Krasheninnikov, A.S. Kukushkin, T.K. Soboleva, Nucl. Fusion 31 (1991) 145.

[8] H.D. Pacher, A.S. Kukushkin, G.W. Pacher, et al., this conf., subm. to J. Nucl. Mater.

[9] A. Roth, "Vacuum Technology”, North-Holland, Amsterdam, 1996, 554pp. 


\section{Figure captions}

Fig. 1. (a) The standard divertor geometry and computational grid used in the ITER modelling [1]. Both the regular plasma grid aligned to the magnetic field and the triangular grid used for neutral transport in the plasma-free areas of model are shown. The PFR interface areas where the neutral pressure is averaged to produce the $p_{D T}$ parameter are indicated; (b) expanded plasma grid for calculations without dome

Fig. 2. $q_{p k}$ (a) and $n_{\text {He_sep }} \cdot S_{\#}$ (b) vs. normalized neutral pressure $\mu$ in PFR for the two "domeless" plasma grid options, compared with the standard ITER case. Power input to the SOL $P_{i n}=100 \mathrm{MW}$. The incipient detachment point on each curve is marked with a big circle and corresponds to neutral pressure at the pump duct $p=9$ Pa for the standard dome case

Fig. 3. (a) Typical profiles of He fluxes into the under-dome channel from the inner and outer divertor legs. X co-ordinate is oriented along the liner from divertor bottom (0) to the dome. Positive values correspond to the flux towards the pump, negative to the backflow; (b) Divertor geometry with a longer dome

Fig. 4. DT neutral influx into the core (a), $q_{p k}$ (b), helium ion density at the separatrix (c) and helium neutral influx to the core (d) vs. $\mu$ for the standard configuration ( $\xi=0.1$ ) and for two long dome options ( $\xi=0.1$ and 0.2 ) 
Figures

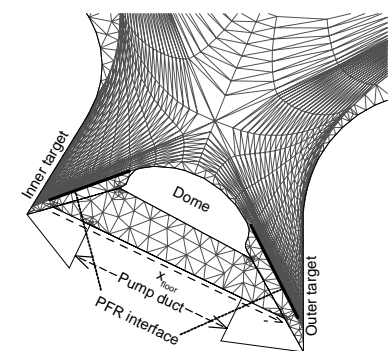

(a)

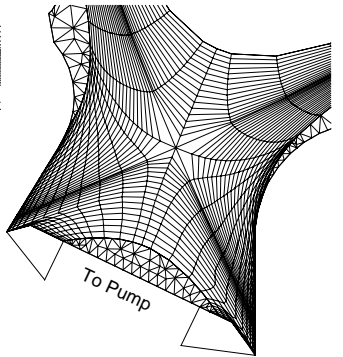

(b)

Fig. 1. 


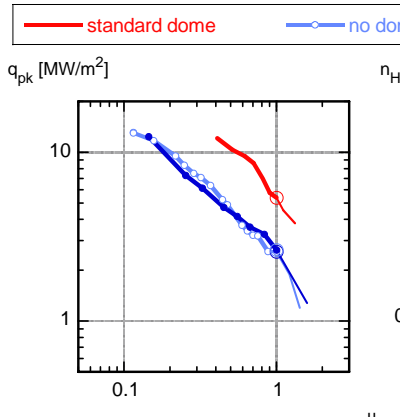

(a)

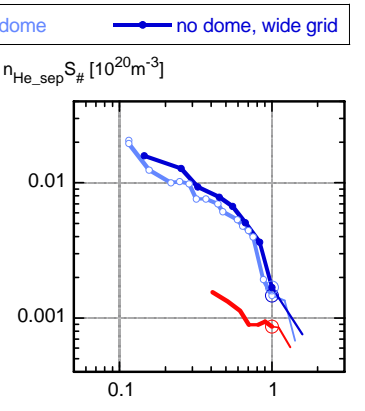

(b)

Fig. 2. 


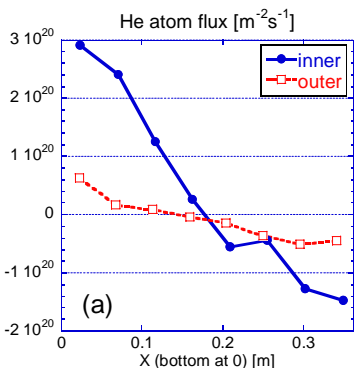

(a)

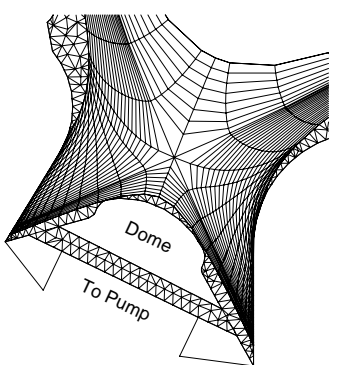

(b)

Fig. 3. 


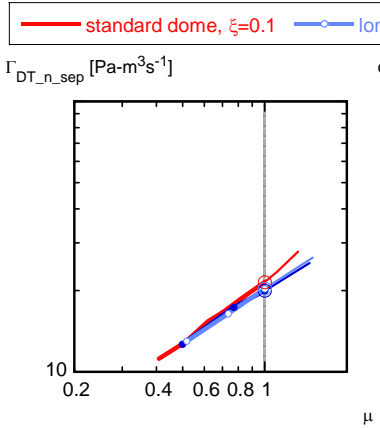

(a)

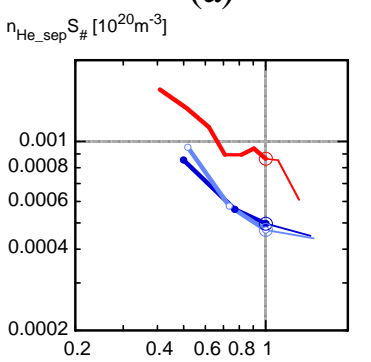

(c)

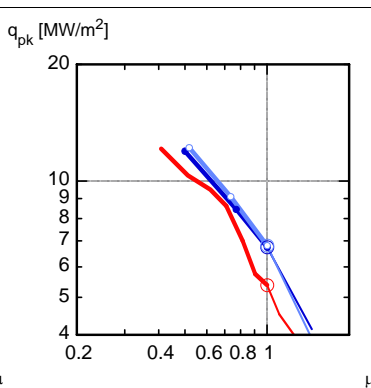

(b)

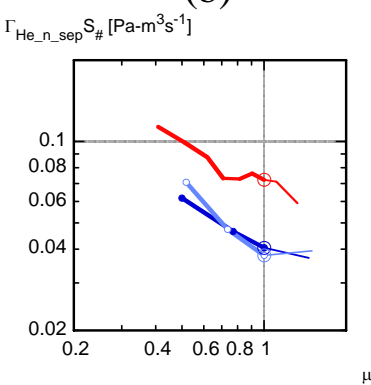

(d)

Fig. 4. 